\title{
BASE DE DATOS ECO-HIDROLOGICA DE LOS RIOS DE CHILE: UNA HERRAMIENTA DE GESTION PARA LOS ECOSISTEMAS ACUATICOS
}

\section{ECO-HYDROLOGICAL DATABASE OF CHILEAN RIVERS: A TOOL FOR MANAGEMENT OF AQUATIC ECOSYSTEM}

\author{
M. Peredo-Parada ${ }^{1 *}$, F. Martínez-Capel ${ }^{1}$, V. Garófano-Gomez ${ }^{1}$, M. Atenas ${ }^{2}$, F. Riestra ${ }^{3}$ \\ ${ }^{1}$ Instituto de Investigación para la Gestión Integrada de Zonas Costeras (IGIC). \\ Universidad Politécnica de Valencia. España. \\ ${ }^{2}$ Departamento de Protección y Conservación de Recursos Hídricos. Dirección General de Aguas. \\ Ministerio de Obras Públicas. Gobierno de Chile. Chile \\ ${ }^{3}$ Unidad de Fiscalización. Dirección General de Aguas. Ministerio de Obras Públicas. Gobierno de Chile. Chile \\ * e-mail:matiasperedo@gmx.net
}

\begin{abstract}
RESUMEN
El conocimiento de las especies nativas de Chile se caracteriza por ser insuficiente, incompleto, fragmentado y poco actualizado, lo cual constituye una de las principales limitantes para desarrollar estrategias de conservación de especies o planes de conservación y recuperación de sistemas acuáticos. Lo anterior cobra mayor importancia dada la mayor presión que tienen actualmente estos ecosistemas en el país. Para paliar este vacío se ha confeccionado una Base de Datos Eco-Hidrológica de los ríos de Chile. Esta base de datos cuenta con información hidrológica, fisicoquímica de las aguas, sedimentológicas e información íctica. Esta última información se ha basado en recopilación bibliográfica de literatura referente a distribución de peces, dividida en tres niveles (I, II y III) de acuerdo al detalle y escala espacial de la información (macroescala, mesoescala y microescala, respectivamente). La Base de Datos Eco-Hidrológica confeccionada la conforman 393 estaciones fluviométricas, 361 estaciones de calidad del agua, 92 estaciones sedimentológicas y un total de 1580 registros de peces distribuidos a lo largo de todo Chile. Esta base de datos se ha integrado en la plataforma de gestión "Clasificación Eco-hidrológica de los ríos de Chile" (REC-Chile), la cual se construyó basándose en los factores medioambientales que controlan el régimen hídrico de un río. La utilización conjunta de la base de datos junto con REC-Chile permitirá desarrollar una mejor planificación y gestión de los ecosistemas acuáticos en Chile.
\end{abstract}

Palabras Claves: Chile, REC-Chile, base de datos eco-hidrológica, gestión ecosistemas acuáticos, peces nativos chilenos.

\begin{abstract}
The limited knowledge of Chilean native species is one of the main constraints to develop conservation strategies for species or conservation planning and rehabilitation of aquatic ecosystems. To fill this gap it was developed an EcoHydrological Database of the Chilean rivers. This database contains information about hydrology, water quality, substrate and fish information. The latter information was based on literature collection concerning on fish distribution, divided into three levels (I, II and III) according to detail and spatial scale of the information (macro, meso and microscale, respectively). The database has 393 hydrological stations, 361 water quality stations, 92 substrate stations and a total of 1580 fish records distributed throughout Chile. This database has been integrated into the management platform «River Environmental Classification of Chilean Rivers» (REC-Chile), which was built based on environmental factors which control the hydrological pattern of a river. The joint use of the database along with REC-Chile will further develop planning and management of aquatic ecosystems in Chile.
\end{abstract}

KeYwords: Chile, REC-Chile, Eco-Hydrological Database, aquatic ecosystems management, Chilean native fish. 


\section{INTRODUCCION}

Las características hidrológicas y morfológicas de Chile han sido los factores claves en la evolución de los peces dulceacuícolas de Chile (Vila et al. 1999). El aislamiento biogeográfico y las características hidrológicas de los ríos son los principales factores que justifican la baja riqueza específica y el alto endemismo junto con la tendencia generalizada a poseer tamaños pequeños (Vila $e t$ al. 1999, Dyer 2000a, Habit et al. 2006, Vila et al. 2006a).

En Chile, la fauna íctica nativa está comprendida por 11 familias, 17 géneros y 44 especies (Dyer 2000a, Habit et al. 2006). Los grupos más representativos corresponden a los órdenes Siluriformes (11 especies), Osmeriformes (9 especies), Atheriniformes (7 especies). También se representan en menor grado los Petromyzontiformes (2 especies), Characiformes (4 especies), Cyprinodontiformes (6 especies), Perciformes (4 especies) y Mugiliformes (1 especie).

La mayor riqueza de especies nativas se encuentra en la zona centro-sur del país entre los ríos Maule e Imperial (Dyer 2000a). El río Biobío es aquél que posee la mayor diversidad íctica con 18 especies nativas (Vila et al. 1999, Dyer 2000a). El extremo norte del país presenta un alto índice de endemismo, con especies que sólo habitan en una cuenca determinada (Vila et al. 1999, Dyer 2000a).

A la riqueza íctica nativa del país hay que añadir 22 especies exóticas, de las cuales sólo dos (Cheirodon interruptus y Odontesthes bonaerensis) están emparentadas a nivel de familia con alguna especie chilena (Dyer 2000a). Entre las especies exóticas, la trucha arcoiris (Oncorhynchus mykiss), trucha café o común (Salmo trutta), salmón del atlántico (Salmo salar) y carpa (Ciprinius carpio) son las más importantes desde el punto de visto económico y de distribución en el país, destacando en el extremo sur del país el predominio de la familia exótica Salmonidae debido a su alto nivel de adaptación a las aguas frías del sur de Chile (Soto et al. 2006). El estado de conservación de los peces nativos de Chile presenta grandes problemas, ya que solo trece especies están consideradas "fuera de peligro". Los taxa con mayor número de especies con problemas en nivel de "peligro de extinción" son los Siluriformes (7 especies) y Cyprinodontiforme (4 especies). Estos dos órdenes coinciden con los de mayor endemismo en cuanto a número de especies y a su limitada distribución geográfica (Vila et al. 1999, Dyer 2000a, Habit et al. 2006)

Dentro de las principales causas del deteriorado estado de conservación de los peces dulceacuícolas de Chile, está la modificación del régimen hidrológico debido a canalizaciones, represamiento de los ríos (Vila et al. 1999) y extracción de agua para riego (Habit et al. 2006), lo cual se traduce en la destrucción del hábitat físico de las especies. La alteración de la calidad fisicoquímica del agua, es otra causa importante del deterioro del hábitat (Vila et al. 1999). Las acciones más comunes son vertidos de residuos industriales líquidos y aguas residuales, extracción de áridos, sustitución de bosque nativo por bosque forestal y contaminación difusa por pesticidas (Habit et al. 2006). Y finalmente, la acción de las especies introducidas en los inicios del siglo XX, principalmente en la zona centro sur de Chile.

Por otro lado, el conocimiento que se tiene de las distintas especies nativas en relación a su distribución, abundancia o densidad, preferencia de hábitat y biología poblacional y reproductiva, es escaso (Habit et al. 2006), incompleto, fragmentado y poco actualizado (Vila 2001). Dicha información está en relación a Siluriformes (Arratia 1983, Pardo 2002, Habit et al. 2003, Habit 2005, Pardo et al. 2005, Scott et al. 2007), Osmeriformes (Peredo \& Sobarzo 1994, Murillo \& Ruiz 2002, Cussac et al. 2004), Atheriniformes (Bahamondes et al. 1979, Comte \& Vila 1987, Dyer 2000b), Perciformes (Habit 1998, Habit \& Belk 2007), Cyprinodontiformes (Vila 2006, Vila et al. 2006b). Recopilación de los estudios realizados a la fecha pueden ser revisados en Habit et al. (2006), Vila $e t$ al. (2006a) y Ruiz et al. (2006). Una completa lista sistemática de las especies puede ser revisada en Dyer (2000a) y en Vila et al. (2006a).

De acuerdo a lo anterior, el escaso conocimiento existente a la fecha no permite avanzar en políticas o gestión para la conservación y/o rehabilitación de ecosistemas acuáticos, como por ejemplo, en el uso de metodologías de simulación del hábitat físico para la determinación del régimen ambiental de caudales (Tharme 2003, Benetti et al. 2004); en la elaboración de directrices en proyectos de rehabilitación fluvial (Bond \& Lake 2003) y en el desarrollo de medidas de mitigación y planes de conservación de las especies dulcacuícolas de Chile (Habit et al. 2006).

Como una medida para paliar estos vacíos de información y avanzar en políticas de conservación y rehabilitación de ecosistemas acuáticos, se ha 
confeccionado una base de datos acoplada a la plataforma de gestión "Clasificación Eco-Hidrológica de los ríos de Chile" (REC-Chile) (Peredo et al. resultados no publicados). La plataforma de gestión REC-Chile es la adaptación a las condiciones ambientales de Chile de la herramienta River Environmental Classification (REC) desarrollada por Snelder \& Biggs (2002). La utilización conjunta de un sistema de clasificación o zonificación, como REC-Chile, y una base de datos de peces constituyen la base para la determinación del estado ecológico de los ríos basado en índices ícticos (Schmutz et al. 2007, Noble et al. 2007), entre otros.

Dentro de las potencialidades de uso que permite la REC están aquellas destinadas al manejo del recurso hídrico a nivel de cuenca (Snelder \& Huguey, 2005), a la caracterización fisicoquímica de los ríos (Snelder et al. 2004a), a la conservación de ecosistemas acuáticos (Peredo et al. resultados no publicados) y a la planificación y gestión medioambiental (Snelder et al. 2004b, Peredo et al . resultados no publicados).

La Clasificación Eco-Hidrológica de los ríos de Chile, es una herramienta que consiste en la superposición jerárquica de las variables o factores ambientales que son los causantes principales de la variación espacial del patrón hidrológico en un río (Snelder \& Biggs 2002, Snelder et al. 2005). Entre los factores que controlan este patrón hidrológico en Chile están el clima, la altitud de la subsubcuenca, presencia de glaciares o lagos, la geología, la posición relativa de la subsubcuenca con respecto a la cuenca en la cual se encuentra el tramo de río, el uso del suelo de la subsubcuenca, la pendiente del tramo de río, etc. De estas variables se definieron los factores controladores aplicables a Chile, los cuales resultaron ser: "Clima", "Origen de flujo" (que engloba las variables pertenecientes a altitud, glaciares, lagos), "Geología", "Posición relativa de subsubcuenca" (definiendo zonas de cabecera, tramos medio y alto y desembocadura) "Uso de suelo" y "Pendiente del tramo del río" (Peredo et al. resultados no publicados).

Los factores controladores que influyen sobre la magnitud y estacionalidad del flujo son el "Clima", "Origen de Flujo" y "Posición relativa de subsubcuenca". Aquellos que tienen mayor influencia sobre la calidad de las aguas son los factores "Geología" y "Uso de suelo", mientras que el factor "Pendiente del tramo de río" influye sobre las condiciones hidráulicas (Snelder and Biggs 2002,
Snelder et al. 2005, Peredo et al. resultados no publicados).

El resultado de la combinación de estos factores controladores es una clasificación única para cada tramo de río, el cual permite ser caracterizado de acuerdo a variables hidrológicas, fisicoquímicas o una combinación de éstas. Los resultados de la aplicación de la REC en Chile ha sido satisfactoria pudiendo caracterizar los tramos de ríos de acuerdo al régimen natural hidrológico, tanto en magnitud como en estacionalidad del hidrograma. (Peredo $e t$ al. resultados no publicados).

En relación a lo anterior, el objetivo del presente trabajo ha sido confeccionar una base de datos georreferenciada que integre información íctica, hidrológica y fisicoquímica de los ríos de Chile, la cual en conjunto con la REC-Chile o algún sistema de clasificación alternativo, puedan servir de base para planificar y gestionar el ecosistema acuático de los ríos de Chile.

\section{MATERIALES Y METODOS}

La base de datos se generó a partir de dos grandes bloques de información. El primer bloque corresponde a información gubernamental proporcionada por la Dirección General de Aguas (DGA) del Ministerio de Obras Públicas (MOP), relativas a fluviometría, calidad del agua y sedimentología. El segundo bloque es información íctica obtenida a partir de recopilación bibliográfica de publicaciones científicas e informes públicos generados de diversos proyectos. En la selección de la literatura se ha dado preferencia a aquella que incluye información de especies nativas.

\section{Información gubernamental.}

La información fluviométrica corresponde a la información de cada estación fluviométrica existente en Chile: Coordenadas UTM, código BNA (código oficial de identificación de la estación), y códigos de cuenca, subcuenca y subsubcuenca en la cual se sitúan. Análogamente, la información fisicoquímica es la información de cada estación de calidad de aguas: coordenadas UTM, código BNA, parámetros fisicoquímicos que se miden en cada una de ellas y la frecuencia a la cual son medidos dichos parámetros. Y por último, para cada estación sedimentológica se dispone de las coordenadas UTM y código BNA. 
Información de fauna íctica.

Dada la heterogeneidad en la escala espacial de la información entregada en la bibliografía especializada e informes de proyectos públicos, se definieron tres niveles (I, II, III) de información de fauna íctica, de acuerdo al detalle de información por parte del autor y a la escala espacial de ésta.

La información del Nivel $\mathrm{I}_{3}$ se define a una escala espacial macro $\left(10^{5}\right.$ a $\left.10^{3} \mathrm{~km}^{2}\right)$. Este nivel de información corresponde a aquel en donde el autor señala distribuciones geográficas de peces que abarcan cuencas e incluso regiones. Aquí se incluyen campos con información de la especie, su rango de distribución, autor y fecha de la publicación .

La información del Nivel II comprende información a nivel de mesoescala, teniendo como escala espacial desde la cuenca hasta un tramo local de río $\left(10^{3}\right.$ a $\left.10^{1} \mathrm{~km}^{2}\right)$. Se ha incluído en este nivel aquella información señalada por el autor(es) referente al río, tramo y/o localidad en donde se practicó el muestreo. Sin embargo, el autor (es) no especifíca mayores detalles sobre el tipo de muestreo utilizado, resultados cuantitativos del muestreo, fecha de realización de éste, entre otros. Los campos utilizados para este nivel contienen información de la especie, fecha de muestreo, año y autor de la publicación, código de la cuenca, subcuenca y/o subsubcuenca de distribución.

La información del Nivel III es una información más detallada actuando a nivel de microescala. El autor(es) señala las coordenadas de la estación de muestreo, fecha de realización, tipo de muestreo y resultados, así como también puede indicar valores de parámetros fisicoquímicos del agua. Para este nivel de información se incluyen campos con información sobre la especie, autor y año de publicación, lugar de muestreo, características del muestreo, parámetros fisicoquímicos del agua y código de cuenca, subcuenca y subsubcuenca.

Para todos los niveles de información señalados anteriormente, se incluyeron campos sobre el código de cuenca, subcuenca y subsubcuenca. Estos códigos son los oficiales asignados por la DGA, permitiendo así que la base de datos pueda estar conectada con la REC-Chile y con información oficial de la DGA.

La totalidad de la información recopilada (fluviométrica, fisicoquímca, sedimentológica e íctica) se implementó en un Sistema de Información Geográfico, con sistemas de coordenadas PSAD 1956 UTM Huso 18S.

\section{RESULTADOS}

Información gubernamental

La base de datos obtenida cuenta con 393 estaciones fluviométricas, 361 estaciones de calidad del agua, 62 estaciones sedimentológicas. Además cuenta con 124 registros de información Nivel I, 450 registros de informacion Nivel II y con 171 estaciones de muestreo específicos de colecta de peces con 1010 registros pertenecientes a la información Nivel III.

La información hidrológica se obtuvo a partir de 393 estaciones fluviométricas. Actualmente sólo 321 están vigentes (Tabla I). Las estaciones fluviométricas que no están vigentes se han incluído en la base de datos, ya que permite realizar un análisis suplementario en aquellos años donde la estación estaba en vigencia. Las estaciones de calidad de aguas y sedimentológicas también se obtuvieron de la información entregada por la DGA con 361 y 62 estaciones respectivamente. Para ambos casos se incluyeron las estaciones suspendidas, (Tabla I).

\section{Información ícitica}

La información Nivel I contiene 120 registros abarcando la totalidad de las especies ícticas nativas del país. La distribución geográfica de esta información abarca todo Chile, desde la cuenca del río Lluta $\left(18^{\circ} \mathrm{S}\right)$ hasta la Tierra del Fuego $\left(55^{\circ} \mathrm{S}\right)$. En este nivel de información se indican las cuencas, y sus códigos asociados, de los extremos norte y sur de distribución definiendo así el rango de distribución para cada especie.

La información Nivel II contiene 450 registros abarcando 37 especies nativas (25 spp. endémicas, 12 spp. nativas) y 6 especies introducidas. Dentro de las especies nativas con más número de registros estan Trichomyterus areolatus (56 registros), Galaxias maculatus (39 registros), Basilichthys australis (27 registros) y Percichthys trucha (27 registros) (Tabla II). Dentro de las introducidas la especie $O$. mykiss con 83 registros es la especie con mayor abundancia. El rango geográfico de este tipo de información va desde del extremo norte de Chile $\left(18^{\circ} \mathrm{S}\right)$ hasta la zona de Aysén $\left(47^{\circ} \mathrm{S}\right)$.

La información Nivel III contiene 171 estaciones de muestreo de peces con 1010 registros correspondientes a 34 especies nativas (7 spp. nativas y $27 \mathrm{spp}$. endémicas) y 8 especies introducidas. Dentro de las especies nativas con mayor número de registros se encuentran $T$. areolatus (128 registros), P. gillissi (80 registros), 
G. maculatus (67 registros) y B. australis con 65 registros (Tabla II). En las especies introducidas aquellas con mayor cantidad de registros son $O$. mykiss (113 registros) y $S$. trutta (94 registros). En este nivel de información se han incluido los puntos de muestreo en los cuales no se han capturado peces ni nativos ni introducidos.

De la Tabla II se observa que las especies T.areolatus, G. maculatus, $P$. gillissi, B. australis y $P$. trucha, son las especies que mayor cantidad de registros tienen al sumar ambos niveles de información (II y III). Las especies distribuidas en la zona norte de Chile, (O. agassii, O. ascotanensis, $O$. chungaraensis, $O$. laucaensis, $O$. parinacotensis, O. piacotensis, $T$. chungaraensis, $T$. laucaensis y $B$. semotilus) tienen una escasa cantidad de registros en ambos niveles de información. Bajo esta misma situación de baja cantidad de registros se encuentran las especies D. chilensis, G. globiceps, H. macrei, $M$. lapicida y $O$. brevianalis. Además existen especies que no tienen información de Nivel II ni tampoco de Nivel III, como son: B. gothei, $C$. killiani, G. alpinus, O. itatanum у O. hatcheri.

La distribución geográfica de las estaciones de muestreo de peces recopilada a nivel de regiones, señalando cuencas involucradas, rango de fecha en el cual se realizaron los muestreos y las referencias de éste se muestran en la Tabla III. De ésta se destaca la heterogeneidad en la densidad de estaciones por muestreo en cada región, existiendo zonas de Chile con mayor densidad (entre las cuencas Rapel e Imperial) y otras con menor densidad (entre las cuencas Loa y Choapa). Al sur de la cuenca del río Bueno no hay estaciones de muestreo.

La utilización conjunta de los tres niveles de información (I, II y III) en la plataforma REC-Chile, permite visualizar por completo el rango de distribución de la especie en estudio. Poniendo como ejemplo la especie Percichthys trucha (Fig. 1), se aprecia que el rango de distribución mostrado por la información Nivel I va desde la cuenca del río Aconcagua hasta el río Maullín (Dyer 2000a). La información Nivel II para esta especie se sitúa dentro del rango definido por Dyer (2000a), abarcando la totalidad de las cuencas Aconcagua, Maipo y Biobío, sin embargo, las cuencas intermedias no muestran información de Nivel II, y sólo en las cuencas Imperial, Toltén, Valdivia y Bueno se aprecia información de la presencia de esta especie en algunas de sus subsubcuencas. Por último, la información de Nivel III se distribuye entre los ríos Mataquito y Valdivia. Se observa también la ausencia de esta especie desde la cuenca del río Rapel hacia el norte.

TABLA I. Distribución geográfica y estado de las estaciones fluviométricas, fisicoquímicas y sedimentológicas pertenecientes a la base de datos.

TABLA I. Geographic distributions and states of hydrologic, physicoquimical and sedimentology stations of database.

\begin{tabular}{|c|c|c|c|c|c|c|}
\hline \multirow[t]{2}{*}{ Región } & \multicolumn{2}{|c|}{$\begin{array}{l}\mathrm{N}^{\circ} \text { Estaciones } \\
\text { fluviométricas }\end{array}$} & \multicolumn{2}{|c|}{$\begin{array}{l}\mathrm{N}^{\circ} \text { Estaciones } \\
\text { fisicoquímicas }\end{array}$} & \multicolumn{2}{|c|}{$\begin{array}{c}\mathrm{N}^{\mathrm{o}} \text { Estaciones } \\
\text { sedimentológicas }\end{array}$} \\
\hline & Vigentes & Suspendidas & Vigentes & Suspendidas & Vigentes & Suspendidas \\
\hline I Región & 5 & 0 & 4 & 0 & 0 & 0 \\
\hline II Región & 33 & 15 & 21 & 37 & 3 & 0 \\
\hline III Región & 29 & 0 & 28 & 0 & 3 & 0 \\
\hline IV Región & 46 & 3 & 54 & 0 & 6 & 7 \\
\hline V Región y RM & 41 & 0 & 68 & 0 & 12 & 2 \\
\hline VI Región & 10 & 0 & 24 & 0 & 1 & 0 \\
\hline VII Región & 36 & 41 & 27 & 0 & 5 & 0 \\
\hline VIII Región & 47 & 0 & 22 & 0 & 10 & 2 \\
\hline IX Región & 29 & 0 & 18 & 0 & 8 & 0 \\
\hline X Región & 45 & 16 & 25 & 33 & 2 & 1 \\
\hline Total & 321 & 72 & 291 & 70 & 50 & 12 \\
\hline
\end{tabular}


Gayana 73(1), 2009

TABLA II. Distribución y números de registros (entre paréntesis) de cada especie nativa por nivel de información (II y III).

TABLA II. Distribution and numbers of measures (in brackets)of each natives species for information level II and III.

\begin{tabular}{|c|c|c|}
\hline \multirow[t]{2}{*}{ ESPECIE } & Nivel II & Nivel III \\
\hline & Distribución & Distribución \\
\hline Aplochiton marinus? Eigenmann 1928 & $\mathrm{~S} / \mathrm{I}$ & $\mathrm{S} / \mathrm{I}$ \\
\hline Aplochiton taeniatus Jenyns 1842 & Chiloé-Aysén (12) & $\mathrm{S} / \mathrm{I}$ \\
\hline Aplochiton zebra Jenyns 1842 & Biobío-Maullín (16) & Itata (1) \\
\hline Basilichthys australis Eigenmann 1928 & Aconcagua-Chiloé (27) & Aconcagua-Valdivia (65) \\
\hline Basilichthys cf. semotilus Cope 1874 & $\mathrm{~S} / \mathrm{I}$ & Loa $(2)$ \\
\hline Basilichthys microlepidotus Jenyns 1841 & Huasco-Aconcagua (4) & Huasco-Choapa (11) \\
\hline Brachygalaxias bullocki Regan 1908 & Biobío-Chiloé (6) & Itata-Valdivia (9) \\
\hline Brachygalaxias gothei Busse 1982 & $\mathrm{~S} / \mathrm{I}$ & $\mathrm{S} / \mathrm{I}$ \\
\hline Bullockia maldonadoi Eiegnmann 1928 & Andalién-Biobío (5) & Andalién-Tolten (36) \\
\hline Cheirodon australe Eigenmann 1928 & Valdivia-Chiloé (3) & Maule-Valdivia (16) \\
\hline Cheirodon galusdae Eigenmann 1928 & Biobío (5) & Maule-Toltén (51) \\
\hline Cheirodon killiani Campos 1982? & $\mathrm{S} / \mathrm{I}$ & $\mathrm{S} / \mathrm{I}$ \\
\hline Cheirodon pisciculus Girard 1855 & Huasco-Biobio (10) & Maipo-Maule (9) \\
\hline Diplomystes camposensis Arratia 1987 & Biobio-Valdivia (2) & Imperial -Valdivia (28) \\
\hline Diplomystes chilensis Molina 1782 & Valdivia (1) & Rapel e Imperial (6) \\
\hline Diplomystes nahuelbutaensis Arratia 1987 & Biobío (6) & Maule-Biobio (9) \\
\hline Galaxias alpinus Jenyns 1842 & $\mathrm{~S} / \mathrm{I}$ & $\mathrm{S} / \mathrm{I}$ \\
\hline Galaxias globiceps Eigenmann 1928 & Maullín-Chiloé (2) & $\mathrm{S} / \mathrm{I}$ \\
\hline Galaxias maculatus Jenyns 1842 & Huasco-Aysén (39) & Itata-Valdivia (67) \\
\hline Galaxias platei Steindachner 1898 & Valdivia-Aysén (11) & Valdivia-Bueno (8) \\
\hline Geotria australis Gray 1851 & Biobío-Aysén (7) & Andalién-Valdivia (38) \\
\hline Hatcheria macrei Girard 1855 & Aysén (2) & \\
\hline Mordacia lapicida Gray 1851 & Biobío (1) & Andalién (7) \\
\hline Mugil cephalus Linnaeus 1758 & Huasco-Valdivia (5) & Andalién (8) \\
\hline Nematogenys inermis Guichenot 1848 & Aconcagua-Biobío (7) & Andalién-Imperial (16) \\
\hline Odontesthes (Cauque) breavianalis Günther 1880 & Choapa-Biobío (3) & $\mathrm{S} / \mathrm{I}$ \\
\hline Odontesthes (Cauque) itatanum? Steindachne 1896 & $\mathrm{~S} / \mathrm{I}$ & $\mathrm{S} / \mathrm{I}$ \\
\hline Odontesthes (Cauque) mauleanum Steindachne 1896 & Biobío-Bueno (14) & Itata-Andalién (3) \\
\hline Odontesthes hatcheri Eigenmann 1909 & $\mathrm{~S} / \mathrm{I}$ & $\mathrm{S} / \mathrm{I}$ \\
\hline Orestias agassi Valenciennes 1846 & Salar Huasco (1) & Salar Huasco, Isluga (3) \\
\hline Orestias ascotanensis Parenti 1984 & Salar Ascotán (1) & S. Ascotan y Carcote (3) \\
\hline Orestias chungaraensis Vila \& Pinto 1986 & Lago Chungara (1) & Lago Chungara (1) \\
\hline Orestias laucaensis Arratia 1982 & Lauca (1) & Río Lauca (1) \\
\hline Orestias parinacotensis Arratia 1982 & Parinacota $(1)$ & Parinacota $(1)$ \\
\hline Orestias piacotensis Vila 2006 & Piacota (1) & Piacota (1) \\
\hline Percichthys melanops Girard 1855 & Aconcagua-Valdivia (5) & Mataquito-Andalién (7) \\
\hline Percichthys trucha Valenciennes 1833 & Aconcagua-Bueno (22) & Mataquito-Valdivia (60) \\
\hline Percilia gillissi Girard 1855 & Maipo-Valdivia (16) & Mataquito-Valdivia (80) \\
\hline Percilia irwini Eigenmann 1928 & Biobio (8) & Maule-Biobío (44) \\
\hline Trichomycterus arelaotus Valenciennes 1840 & Huasco-Chiloé (56) & Aconcagua-Bueno (128) \\
\hline Trichomycterus chiltoni Eigenmann 1928 & Aconcagua-Biobio (5) & Maule-Biobio (9) \\
\hline Trichomycterus chungaraensis Arratia 1983 & Lago Chungara (1) & Lago Chungara (1) \\
\hline Trichomycterus laucaensis Arratia 1983 & Río Lauca (1) & Lauca y Parinacota (2) \\
\hline Trichomycterus rivulatus Valenciennes 1840 & Salar Huasco (1) & Altiplánicas (4) \\
\hline
\end{tabular}

S/I: Sin Información. No information 
Base eco-hidrológica de los ríos de chile: Peredo-Parada, M. ET AL.

TABLA III. Número de estaciones de muestreo de peces recopiladas en la información de Nivel III y agrupadas de acuerdo a región y cuenca.

TABLA III. Number of fish sampling stations compiled in information level III and grouped by region and basin.

\begin{tabular}{|c|c|c|c|c|}
\hline Región & Cuenca & $\begin{array}{c}\mathrm{N}^{\mathrm{o}} \\
\text { estaciones }\end{array}$ & $\begin{array}{l}\text { fechas de } \\
\text { muestreos }\end{array}$ & Referencias \\
\hline Región I de & Altiplánica & 8 & 2006 & U. de Chile Lab. Limnologia \\
\hline Tarapacá & Qbda Camarones & 1 & 2006 & U. de Chile Lab. Limnologia \\
\hline Región II de & Rio Loa & 4 & 2006 & U. de Chile Lab. Limnologia \\
\hline Antofagasta & Salar Michinca & 2 & 2006 & U. de Chile Lab. Limnologia \\
\hline Región III de & Rio Copiapo & 2 & 2006 & U. de Chile Lab. Limnologia \\
\hline Copiapó & Rio Huasco & 4 & 2006 & U. de Chile Lab. Limnologia \\
\hline $\begin{array}{l}\text { Región IV de } \\
\text { Coquimbo }\end{array}$ & Rio Choapa & 1 & $1982-1984$ & Comte et al. \\
\hline $\begin{array}{l}\text { Región V de } \\
\text { Aconcagua y }\end{array}$ & Rio Aconcagua & 9 & $2001-2006$ & CADE IDEPE, EDIC. \\
\hline $\begin{array}{l}\text { Región } \\
\text { Metropolitana }\end{array}$ & Costeras (Maipo) & 4 & 2007 & Fernández \\
\hline $\begin{array}{l}\text { Región VI del } \\
\text { Gral. O'higgins }\end{array}$ & Rio Rapel & 21 & $2002-2006$ & $\begin{array}{l}\text { M\&P Ltda, Conc. Convento } \\
\text { Viejo, Geotecnica. }\end{array}$ \\
\hline Región VII del & Rio Mataquito & 12 & $2000-2006$ & DGA, Est. y Gest. Ambiental \\
\hline Maule & Rio Maule & 21 & $2000-2006$ & $\begin{array}{l}\text { A\&C Cons. Gesam Cons., } \\
\text { Ingendesa, }\end{array}$ \\
\hline Región VIII de & Rio Itata & 10 & $1993-2000$ & Habit, DGA \\
\hline Biobío & Rio Biobio & 9 & 2001-2003 & Habit \\
\hline & Rio Andalien & 17 & $1986-2000$ & Habit, Ruiz \\
\hline Región IX de la & Rio Imperial & 22 & $1997-2003$ & Campos, DGA, Gutierrez \\
\hline Araucanía & Rio Tolten & 5 & 1997 & Campos \\
\hline Región X de los & Rio Valdivia & 7 & 2005-2006 & EULA \\
\hline Lagos & Rio Bueno & 9 & 2003-2005 & Velasco Asoc. \\
\hline
\end{tabular}

\section{DISCUSION}

La información del Nivel I es la más abundante en extension geográfica, pero también es aquella con mayor incertidumbre, puesto que para una misma especie diversos autores difieren en el rango de distribución, por ejemplo, para Nematogenys inermis, Campos et al. (1993) señalan que está distribuida entre los ríos Rapel y Biobío, pero Dyer (2000a) señala que la distribución corresponde entre los ríos Maipo y Biobío. Otro ejemplo sucede con Percilia gillisi a la cual Vila et al. (1999) la distribuye entre los ríos Maipo a Maullín, pero Dyer (2000a) extiende esa distribución por el norte hasta la cuenca del Aconcagua. A esta incertidumbre de la distribución de la especie se le debe agregar el hecho que bajo este nivel de información no se puede deducir sectores específicos de presencia o distribución de una especie dentro de una cuenca, por tanto, es imposible indicar si la especie se ubica preferentemente en zona de desembocadura o de cabecera, etc., dentro de una cuenca. En consecuencia, se asume que la especie se distribuye en toda cuenca dentro del rango geográfico definido para ésta.

La información íctica del Nivel II trabaja a una escala menor que el nivel I, por lo cual la precisión en la 
ubicación, distribución y preferencias de hábitat de la especie es mayor, pudiendo así incorporar 450 registros para este nivel. La escala espacial mínima de distribución viene dada por la subsubcuenca, por tanto, se supondrá que la especie se distribuye en toda la subsubcuenca (como mínimo) a pesar de que el autor la haya señalado sólo para una localidad determinada. Dado el tipo de información perteneciente a este nivel, se puede deducir la presencia de una especie, pero no su ausencia, puesto que sólo se indica la subsubcuenca, subcuenca y/o cuenca en la cual se muestreó la especie, pero no en las que la especie está ausente.

La información del Nivel III define el nivel más completo de la base de datos, contiene 171 estaciones de muestreo con 1010 registros. Como en cada estación de muestreo se indica la totalidad de las especies obtenidas en las campañas de muestreo, este nivel no sólo permite determinar presencia de una especie sino también la ausencia de ésta. El rango geográfico de este nivel es el más limitado de los tres niveles, definiéndose entre las cuencas del río Lluta y la cuenca del río Bueno. Hacia el sur de éste no hay datos.

En la zona Norte de Chile ( $\left.18^{\circ}-26^{\circ} \mathrm{S}\right)$, la información es escasa y se traduce en algunos puntos tomados en sectores altiplánicos y a lo largo de río Loa. En la zona denominada el Norte Chico $\left(26^{\circ}-31^{\circ} \mathrm{S}\right)$, los puntos de muestreos son heterogéneamente distribuidos, los ríos Huasco y Copiapó cuentan con información reciente, sin embargo, los muestreos en el río Choapa datan de 1987. Más preocupante son los casos de los ríos Elqui y Limarí que no presentan información de Nivel III.

La zona Central de Chile $\left(31^{\circ}-39^{\circ} \mathrm{S}\right)$ tiene una mayor densidad de información por cuenca, sin embargo, existen cuencas que no presentan puntos de muestreo, como es el caso de los ríos Maipo, Mapocho y Cachapoal.

En la zona Sur $\left(39^{\circ}-42^{\circ} \mathrm{S}\right)$, la densidad disminuye drásticamente, distinguiéndose dos zonas. La primera de ellas, comprendida entre los ríos Imperial y Bueno, presenta una buena cantidad de información, a excepción del río Toltén que presentan una baja densidad de puntos y no presenta información actualizada. La segunda zona se ubica desde la cuenca del Río Maullín hacia el sur, en donde la cantidad de información de Nivel III es nula.

Es importante destacar que existen cuencas en las cuales no existe información científica disponible de Nivel III, como por ejemplo, en las cuencas del río Aconcagua, Maipo, Rapel, Maule, etc (Tabla II). La información disponible en estas cuencas es información procedentes de proyectos de ingeniería que según la Ley 19.300 deben someterse al Sistema de Evaluación de Impacto Ambiental.

La cantidad de registros de información en los niveles II y III para cada especie va acorde al estado de conservación de las especies definido en Habit et al. (2006). Las especies consideradas como "vulnerable" presentan una mayor cantidad de registros y aquellas con un alto endemismo y/o consideradas como en "Peligro de extinción" o "Indeterminadas" tienen escasa cantidad de registros. El género Orestias es un claro ejemplo, ya que su marcado endemismo va acorde a una baja cantidad de registros. De la misma forma, las especies distribuidas en el norte de Chile (B. semotilus, T. chungaraensis y T. laucaensis) y las distribuidas en el extremo sur (H. macrei y $G$. globiceps) también presentan baja cantidad de registros. Un caso especial lo presenta la especie $O$. brevianalis que está considerada como vulnerable y el rango de distribución comprende las cuencas Elqui y Chiloé (Dyer 2000a), sin embargo, sólo presenta registros en la información del Nivel II. Esto quiere decir que al no presentar registros en la información de Nivel III ningún autor ha encontrado esta especie en los muestreos ubicados dentro del rango de distribución. Por tanto, podría sugerirse reconsiderar el estado de conservación de esta especie.

La utilización de los tres niveles de información permite aprovechar la escasa información dulceacuícola existente, pudiendo utilizarse como complementarios o en su caso para redelimitar la distribución de una especie. Para el caso ilustrado en la Fig. 1, se indica que el rango de distribución de la especie $P$. trucha definida por los niveles I y II está comprendido entre los ríos Aconcagua y Maullín; sin embargo la información de Nivel III señala que se distribuye entre los ríos Mataquito y Valdivia, presentando una ausencia hacia el norte del río Rapel, lo cual contradice al rango de distribución definido en la literatura (Campos et al. 1993, Vila et al. 1999, Dyer 2000a). Si se considera que los muestreos en las cuencas Aconcagua y Maipo son recientes, se podría deducir que el rango de distribución para este especie está en retroceso. La inclusión de información hidrológica y fisicoquímica a la base de datos expande las posibilidades de uso de esta base de datos, permitiendo abordar los problemas y sus soluciones 
de una manera más holísitica. Además, al incorporar las estaciones fluviométricas y fisicoquímicas que están actualmente suspendidas, permitiría conocer el estado anterior de la calidad de las aguas en los ríos y eventualmente estos niveles podrían ser usados como niveles de referencia en posteriores estudios.

Se entrega, ante la comunidad científica, una base de datos ecohidrológica confeccionada a partir de la recopilación de información hidrológica, fisicoquímica e íctica que contiene información de diversa escala espacial. Esta base de datos se implementó en un Sistema de Información Geográfico, con sistemas de coordenadas PSAD 1956 UTM Huso 18 S.

Esta base de datos está acoplada a la plataforma de gestión «Clasificación Eco-Hidrológica de los ríos de Chile» (REC-Chile), transformándose así, en una herramienta eficaz para sentar las bases de proyectos de gestión, planificación y conservación del medio acuático.

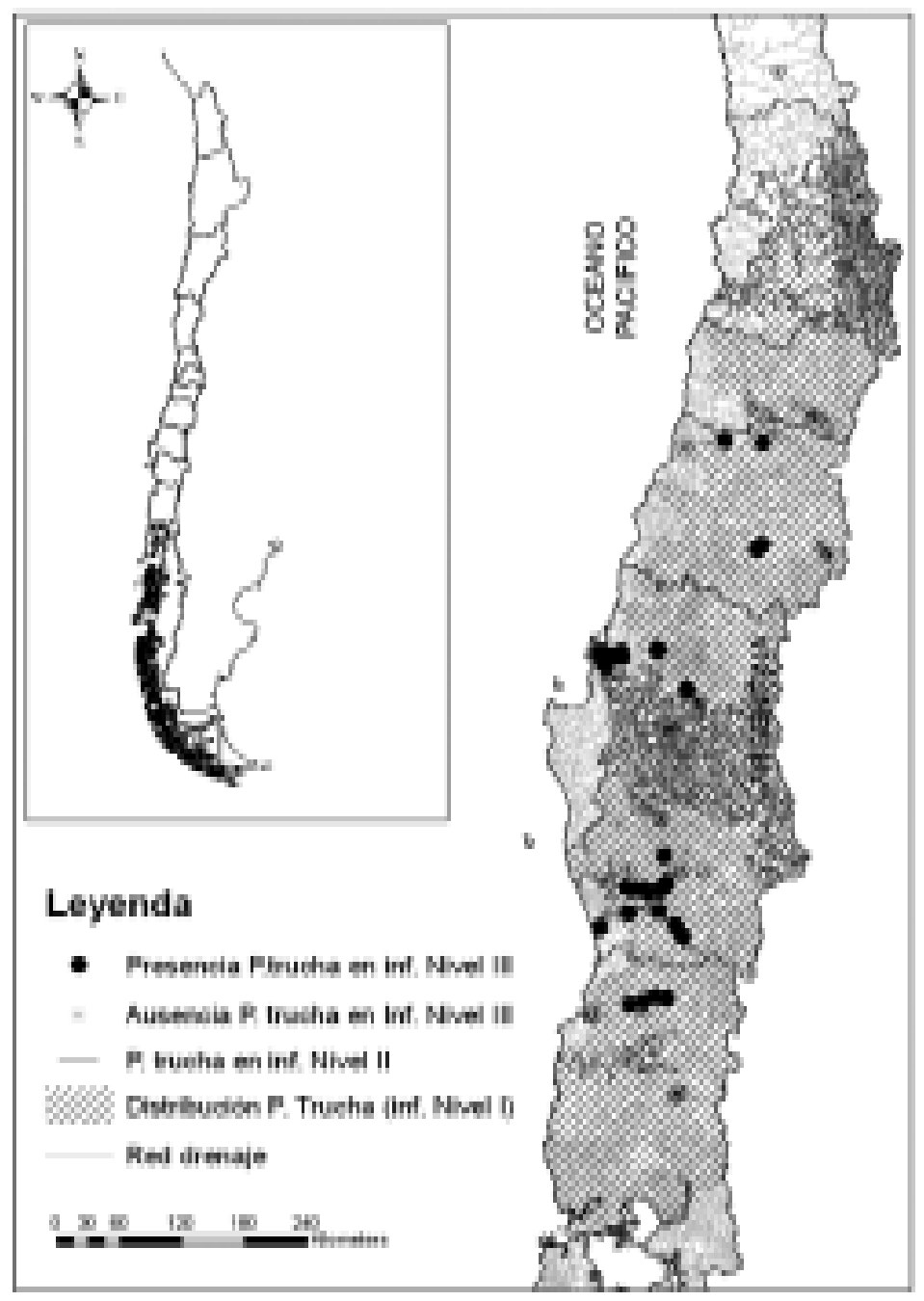

FiguRA 1. Representación gráfica de los Niveles de información I, II y III para la especie Percichthys trucha

FIGURE 1. Graphic representation of the information levels I, II and III for the species Percichthys trucha 


\section{AGRADECIMIENTOS}

Se agradece a CONICYT a la Agencia Española de Cooperación Internación para el desarrollo (AECID) por el apoyo financiero otorgado a Matías Peredo y a Mónica Musalem por los servicios prestados en la DGA.

\section{BIBLIOGRAFIA}

Arratia, G. 1983. Preferencias de hábitat de peces siluriformes de aguas continentales de Chile (Fam. Diplomystidae y Trichomycteridae). Studies on Neotropical Fauna and Environment 18 (4): 217 237.

Bahamondes, I., D. Soto \& I. Vila. 1979. Hábitos alimentarios de los pejerreyes (Pisce:Atherinidae) del Embalse Rapel. Medio Ambiente 4 (1): 3-18.

Benetti, A.D., A.E. Lanna \& M.S. Colbachini. 2004. Current practices for establishing environmental flows in Brazil. River Research and Applications 20: 427-444.

Bond, N.R. \& P.s. LAKE. 2003. Characterizing fish-habitat associations in streams as the first step in ecological restoration. Austral Ecology 28: 611621.

Campos, H., V. Ruiz \& J.f. Gavilán. 1993 Peces del río Biobío. Publicaciones de divulgación. EULA 5. 100pp.

Comte, S. \& I. Vila. 1987. Modalidad reproductiva de Basililchthys microlepidotus (Jenyns), en el río Choapa (Pisces:Atherinidae). Anales Museo de Historia Natural, Valparaíso 18: 85-94.

Cussac, V., S. Ortubay, G. Iglesias, D. Milano, M. Lattuca, J. Barriga, M. Battini \& M. Gross. 2004. The distribution of South American galaxiid fishes: the role of biological traits and post-glacial history. Journal of Biogeography 31: 103-121.

DYER, B. 2000A. Revisión sistemática y biogeográfica de los peces dulceacuícolas de Chile. Estudios Oceanológicos, Antofagasta 19: 77-98.

DYER, B. 2000B. Revisión sistemática de los pejerreyes de Chile (TELEOSTEI, ATHERINIFORMES). Estudios Oceanológicos, Antofagasta 19: 99-127.

Habit, E. 1998. Análisis de la dieta de Percilia gillissi (Pisces: Percilidae) en poblaciones del río y canales de riego (Cuenca del Itata, VIII Región) Theoria 7: 33-46.

HaBit, E. 2005. Aspectos de la biología y hábitat de un pez endémico de Chile en peligro de extinción (Diplomystes nahuelbutaensis Arratia, 1987). Interciencia 30 (1): 8-11.

Habit, E. \& M. BelK. 2007. Threatened fishes of the world: Percilia irwini (Eigenmann 1927) (Perciliidae). Environmental Biology of Fishes 78: 213-214.

Habit, E., P. Victoriano \& H. Campos. 2003. Ecología trófica $y$ aspectos reproductivos de
Trichomycterus areolatus (pisces, Trichomycteridae) en ambientes lóticos artificiales. Revista de Biología Tropical 53 (12): $195-210$.

Habit, E., B. Dyer \& I. Vila. 2006. Estado de conocimiento de los peces dulceacuícolas de Chile. Gayana 70 (1): 100-112.

Murillo, V. \& V.H. Ruiz. 2002. El puye Galaxias globiceps EIGENMANN 1927 (Osteichthyes: Galaxiidae): ¿Una especie en peligro de extinción?. Gayana 66 (2): 191-197.

Noble, R., R. Cowx, D. Goffaux \& P. Kestemont. 2007. Assessing the health of European rivers using functional ecological guilds of fish communities: standardising species classification and approaches to metric selection. Fisheries Management and Ecology 14: 381-392.

Pardo, P. 2002. Diferenciación morfológica de poblaciones de Trichomycterus areolatus Valenciennes 1846 (Pisces:Siluriformes: Trichomycteridae) de Chile. Gayana.66 (2): 203 205.

Pardo, R., S. Scott \& I. Vila. 2005. Análisis de formas en especies chilenas del género Trichomycterus (Osteichthyes: Siluriformes) utilizando morfometría geométrica. Gayana 69 (1): 180-183.

Peredo, S. \& C. Sobarzo. 1994. Actividad gonádica estacional de Galaxias maculatus (Jenyns, 1842) en el río Cautín IX Región, Chile. Boletín Sociedad Biología, Concepción 65: 65-70.

Ruiz, V.H., R. Figueroa \& B. Neira 2006. Índice bibliográfico sobre peces de aguas continentales y estuarinas de Chile. Boletín Sociedad Biología de Concepción 77: 73-110.

Schumtz, S., I. Cowx, G. Haidvogl \& D. Pont. 2007. Fishbased methods for assessing European running waters: a synthesis. Fisheries Management and Ecology 14:369-380.

ScOtT, S., R. PARDO \& I. VILA. 2007. Trophic niche overlap between two Chilean endemic species of Trichomycterus (Teleostei: Siluriformes). Revista Chilena de Historia Natural 80 (4): 431-437

Snelder, T. \& B. Biggs. 2002 Multiscale river environment classification for water resources management. Journal of the American Water Resources Association 38 (5): 1225-1239.

Snelder, T. \& K.F.D. Hughey. 2005. The use of an Ecological Classification to improve Water Resource Planning in New Zealand. Environmental Management 36 (5): 741-756.

SNelder, T., B. Biggs \& M. Weatherhead. 2004a. Nutrient concentration criteria and characterization of patterns in trophic state for rivers in heterogeneous landscapes. Journal of the American Water Resources Association 40 (1): 1-14.

Snelder, T., F. Cattanéo, A. Suren \& B. Biggs. 2004B. Is the River Environment Classification an improved landscape-scale classification of rivers?. Journal of North American Benthological Society 23 (3): 580-598.

SNeLdER, T. B. BigGS \& R. Woods. 2005. Improved eco- 
Base eco-hidrológica de los ríos de chile: Peredo-ParadA, M. ET AL.

hidrological classifications of river. River Research and Applications 21: 609-628.

Soto, D., I. Arismendi, J. Gonzalez, J. Sanzana, F. Jara, C. Jara, E. Guzman \& A. Lara. 2006. Southern Chile, trout and salmon country: invasion patterns and threats for native species. Revista Chilena de Historia Natural 79 (1): 97-117.

THARME, R.E. 2003. A gobal perspective on environmental flow assessment: emerging trends in the development and application of environmental flow methodologies for rivers. River Research and Applications 19: 397-441.

VILA, I. 2001. LA DIVERSIDAD ACUÁTICA: estructura, funciones y salud de las cuencas, bases para el Manejo Integrado en Sustentabilidad, Proyecto PMRH, Ministerio de Obras Públicas, Gobierno de Chile.
VILA,, I. 2006. A new species of killifish en the genus Orestias (Teleostei: Cyprinodontidae) from the Southern high Andes, Chile. Copeia 3: 472-477.

Vila, I., L. Fuentes \& M. Contreras. 1999. Peces límnicos de Chile. Boletín Museo de Historia Natural, Chile. 48: 61-75.

Vila I., R. Pardo, B. Dyer \& E. Habit. 2006a. Peces límnicos: Diversidad, origen y estado de conservación. En: Vila I, A Veloso, R Schlater \& C Ramirez (eds.) Macrófitas y vertebrados de los sistemas límnicos de Chile: 73-103. Editorial Universitaria, Stgo, Chile.

Vila, I., M. Mendez, S. Scott, P. Morales \& E. Poulin. 2006в. Threatened fishes of the world: Orestias ascotanensis Parenti, 1984 (Cyprinodontidae). Environmental Biology Fisheries 80: 491-492.

Recibido: 19.07 .08

Aceptado: 22.05.09 